\title{
Chaotic behavior and its control in a two parameter map with variable Jacobian
}

\author{
Hemanta Kr. Sarmah \\ Department of Mathematics \\ Gauhati University, Guwahati \\ Guwahati, India
}

\author{
Ranu Paul \\ Department of Mathematics \\ Pandu College, Guwahati, \\ Guwahati , India
}

\begin{abstract}
Inthe present paper we have considered the map $W(x, y)=\left(y, a y+b-x^{2}\right)$ where $\boldsymbol{a}, \boldsymbol{b}$ are parameters. The map was originally proposed by Maynard Smith [17] for study of population growth. We have shown how chaos creep into the model. We have used the techniques of Lyapunov exponent, time series analysis, Fourier spectra, Bifurcation diagram, correlation and embedding dimension etc. to draw our conclusions. Further, we have shown how the 'periodic proportional pulse' method can be used to control the chaos generated in the system.
\end{abstract}

Keywords: Chaos, Lyapunov exponent, Bifurcation diagram, Chaos control, Correlation dimension, Embedding dimension

\section{INTRODUCTION}

Over the past few decades, following the discovery of chaotic behavior in deterministic nonlinear dynamical systems, there has been considerable interest in the study of nonlinear differential equations and nonlinear difference equations with applications ranging across physics, chemistry, biology, economics etc. In many of these studies a knowledge of the properties of simple one and two dimensional maps such as the logistic map, the dissipative standard map, the Lorentz map, the Henon map, the tent map, the quadratic map etc. has provided fundamental insights $[1,2,3,8,9,16]$. This has been particularly the case in understanding deterministic chaos, understanding various routes for transitions to chaos as a control parameter is varied, and identifying universality classes for the onset of chaos similar to universality in the theory of critical phenomena.

It is well known that discrete models exhibit chaos more readily than their continuous counterparts. For instance, chaotic dynamics is possible for discrete models of even a single species, but require at least three variables in continuous time.

Chaotic behavior, sometimes, is a desirable result, for example in the case of random number generator, fluid mixing etc. Often, however, it is not. An erratically oscillating machine may cause significant damage to itself and surrounding infrastructure. Moreover, since the chaotic region for a system may contain many possible orbits, being able to select between several of these by changing control parameters allows multiple responses from the same system. Thus, it is oftenuseful to stabilize a chaotic orbit to one that is regular and predictable. Chaos control explores the intrinsic richness of the chaotic behavior by stabilizing unstable periodic orbits embedded in a chaotic attractor.

\section{THE MAP :}

Discrete models of density-dependent population growth have attracted much attention as examples of simple dynamical systems displaying extremely complicated dynamics. These simple models describe the growth of a population with nonoverlapping generations by an equation of the form $x_{n+1}=$ $f\left(x_{n}\right)$, where $x_{n}$ is the population size at time $n$ and $f$ is some suitably chosen function. This leads naturally to the study of maps of an interval into itself and, following the work of $\mathrm{Li}$ and Yorke [25] and May [20] families of one dimensional maps with a single turning point have been extensively studied.

Generalizations of these models take the form of higher dimensional mappings. Notable examples in two dimensions being the density-dependent Leslie models of Guckenheimer et al. [10], and the delayed logistic equation considered by Aronson et al. [4] to name a few.

We consider the $\operatorname{map} W(x, y)=\left(y, a y+b-x^{2}\right)$ wherea, $b$ are parameters.

The model was originally proposed by M. J. Smith [17] who derived the following relationship for the growth of a species with egg and adult age classes $(n+1)=\operatorname{ax}(n)+$ $b x(n-\tau)-c x^{2}(n-\tau)$. Here $x(n)$ denotes the size of the adult population at time ; $\tau$ is the time taken for an egg to develop into an adult; $a \in(0,1)$ is the probability that an adult at timen survives to timen $+1 ; b>0$ is the number of eggs laid per adult per unit time which in optimal conditions (i.e. low $x(n)$ ) survive to become adults; and $c>0$ represents a density-dependent constraint on the fecundity of the adults. In fact $c$ is simply scaling factor which is removed by the change of scale $\rightarrow x / c$, so that

$$
x(n+1)=a x(n)+b x(n-\tau)-x^{2}(n-\tau)
$$

We introduce new variables

$$
x_{1}(n)=x(n-\tau), x_{2}(n)=x(n-\tau+1), \quad \ldots, \quad x_{\tau+1}=x(n)
$$

to obtain, in the usual way, a map of the phase space sending $x_{i}(n) \operatorname{to} x_{i}(n+1), 1 \leq i \leq \tau+1$

$$
\begin{aligned}
& F: R^{\tau+1} \rightarrow R^{\tau+1} \\
& F:\left(x_{1}, x_{2}, \ldots, x_{\tau+1}\right)=\left(x_{2}, \ldots, x_{\tau+1}, a x_{\tau+1}+b x_{1}-x_{1}{ }^{2}\right)
\end{aligned}
$$

If $\tau=0$, this gives the function $F(x)=(a+b) x-x^{2}$ which is equivalent, by a linear coordinate change, to the quadratic 
family $f_{\mu}(x)=\mu x(1-x)$ considered by May [17] as a simple population model.

Our interest is in the case $\tau=1$, where we have

$F\left(x_{1}, x_{2}\right)=\left(x_{2}, a x_{2}+b x_{1}-x_{1}^{2}\right)$,

or after a translation of coordinates

$x_{i} \rightarrow x_{i}-b / 2 \quad F\left(x_{1}, x_{2}\right)=\left(x_{2}, a x_{2}+\tilde{b}-x_{1}^{2}\right)$ where

$\tilde{b}=\frac{a b}{2}+\frac{b^{2}}{4}-\frac{b}{2}$

Renaming the coordinates $x_{1}=x, x_{2}=y$ and also by writing $\tilde{b}=b$, we obtain the map we shall study in detail, viz.

$$
F_{(a, b)}(x, y)=\left(y, a y+b-x^{2}\right)
$$

We will simply write $F$, instead of $F_{(a, b)}$ remembering that the behavior of the map will depend on the parameters $a$ and $b$.

We will investigate the behavior of this map by fixing one parameter and varying the other one.

\subsection{Further Reason for the parameter a to have the largest value 1 :}

The map can be written as $x_{n+1}=y_{n} y_{n+1}=a y_{n}+b-x_{n}^{2}$

So, $\quad y_{n+1}-x_{n+1}=(a-1) y_{n}+b-x_{n}^{2}=h\left(x_{n}, y_{n}\right)$

For extreme values of $h(x, y)$ we must have $h_{x}(x, y)=0$ and $h_{y}(x, y)=0$ from which we get the two conditions $x=0$ and $a=1$. Thus to have an extreme value of the difference of the 2 nd population and 1 st population we must have $a=1$ and then the point of extremum becomes the point of maxima. The maximum value of the difference of population is calculated to be equal to $b$. Since maximum difference is obtained at $a=1$, so a boundary crisis occurs at this parameter value and the trajectories goes off towards $-\infty$. The boundary crisis can be verified by the bifurcation diagram which suddenly ends at $a=1$ and also by the occurrence of overflow in the calculation of iterative values of the populations. So in our following discussions we will vary $a$ from 0 to 1 and choose $b=0.6$.

\subsection{Range for dissipative, conservative and area expanding case :}

The Jacobian matrix is $J_{1}=\left(\begin{array}{cc}0 & 1 \\ -2 x & a\end{array}\right)$. So, Det $J_{1}=2 x$, a variable. The fixed points of the map are given by the equations $y=x$ and $a y+b-x^{2}=y$. So, the fixed points are given by $x_{1,2}^{*}=\frac{a-1 \pm \sqrt{(1-a)^{2}+4 b}}{2}$

Thus the fixed points are $\left(x_{1}^{*}, x_{1}^{*}\right)$ and $\left(x_{2}^{*}, x_{2}^{*}\right)$ and the map has no fixed point if

$b<-\frac{1}{4}(1-a)^{2}$.

For dissipative, conservative and area expanding cases we must have $\mid$ Det $J_{1}|<1$,$| Det J_{1} \mid=1$ and $\mid$ Det $J_{1} \mid>1$ at the fixed point respectively.
That is, the map is dissipative if $|x|<\frac{1}{2}$, conservative if $|x|=\frac{1}{2}$ and area expanding if $|x|>\frac{1}{2}$. Thus the map can be dissipative, conservative or area expanding depending on the initial condition or starting point.

Again the map is dissipative when $\mid$ Det $J_{1} \mid$ at the two fixed points $\left(x_{1}^{*}, x_{1}^{*}\right)$ and $\left(x_{2}^{*}, x_{2}^{*}\right)$ are also less than $\frac{1}{2}$, which gives two condition $a<0.3$ or $a>1.7$. But since the largest value of the parameter for the map is $a=1$ so we need to discard the condition $a>1.7$. Thus the map is dissipative when the magnitude of the first species is less than $\frac{1}{2}$ and the probability of an adult at time $n$ to survive at time $n+1$ is less than 0.3 .

Similarly the map is conservative if the magnitude of the first species is equal to $\frac{1}{2}$ and the probability of an adult at time $n$ to survive at timen +1 is equal to 0.3 , area expanding if the magnitude of the first species is greater $\operatorname{than} \frac{1}{2}$ and the probability of an adult at timen to survive at timen +1 lies in the interval $(3,1]$.

\subsection{Regions in which the fixed points are stable, unstable or saddle :}

Here $J_{1}=\left(\begin{array}{cc}0 & 1 \\ -2 x & a\end{array}\right) . \operatorname{SoTr}\left(J_{1}\right)=a, \operatorname{Det}\left(J_{1}\right)=2 x$.

Fixed points $\operatorname{are}\left(x_{1}^{*}, x_{1}^{*}\right) \operatorname{and}\left(x_{2}^{*}, x_{2}^{*}\right)$ where

$$
x_{1,2}^{*}=\frac{a-1 \pm \sqrt{(1-a)^{2}+4 b}}{2} .
$$

There is no fixed point if $b<-\frac{1}{4}(1-a)^{2} \quad$ A fixed point is

stable if $\left|\operatorname{Tr}\left(J_{1}\right)\right|<1+\operatorname{Det}\left(J_{1}\right)<2$,

unstable if $\left|\operatorname{Tr}\left(J_{1}\right)\right|<\left|1+\operatorname{Det}\left(J_{1}\right)\right|,\left|\operatorname{Det}\left(J_{1}\right)\right|>1$

and saddle if

$$
\left|\operatorname{Tr}\left(J_{1}\right)\right|>\left|1+\operatorname{Det}\left(J_{1}\right)\right|,-\operatorname{Tr}\left(J_{1}^{2}\right)-4 \operatorname{Det}\left(J_{1}\right)>0
$$

at the fixed point .

The fixed $\operatorname{point}\left(x_{1}^{*}, x_{1}^{*}\right)$ is stable when $a<0.3$, unstable if $a>0.3$ but is never a saddle fixed point because otherwise it will lead to an inequality $\sqrt{(1-a)^{2}+4 b}<0$, which is absurd for our chosen set of parameter values.

Similarly the fixed point $\left(x_{2}^{*}, x_{2}^{*}\right)$ is unstable if $a(3 a+2)<$ 3.4 , saddle if $a(3 a+2)>3.4$ but is never a stable fixed point because in this case also we get an absurd inequality.

\section{EXPONENTIAL DIVERGENCE IN THE TRAJECTORIES FOR SLIGHTLY DIFFERENT INITIAL CONDITIONS:}

To show that the map we have considered is sensitively dependent on initial conditions, we plot two trajectories (xtime series) for two slightly different initial conditions. For the first trajectory we have taken the initial condition as $x(0)=0.5, y(0)=0$ (the continuous picture) and for the second one we have taken $x^{1}(0)=0.5+10^{-5}, y^{1}(0)=0+$ $10^{-5}$ (the dotted picture). We have drawn the picture for $b=$ 0.6 and $a=1$ As in dynamical system, we are interested in the long term behavior, we have depicted the trajectories after large number of iterations, neglecting the first 2450 iterations. The figure shows the two trajectories which starts from almost same points diverge exponentially. 
This verifies that the map under consideration is sensitive to the initial conditions and hence there is every possibility that the map may have chaotic behavior for some parameter values.

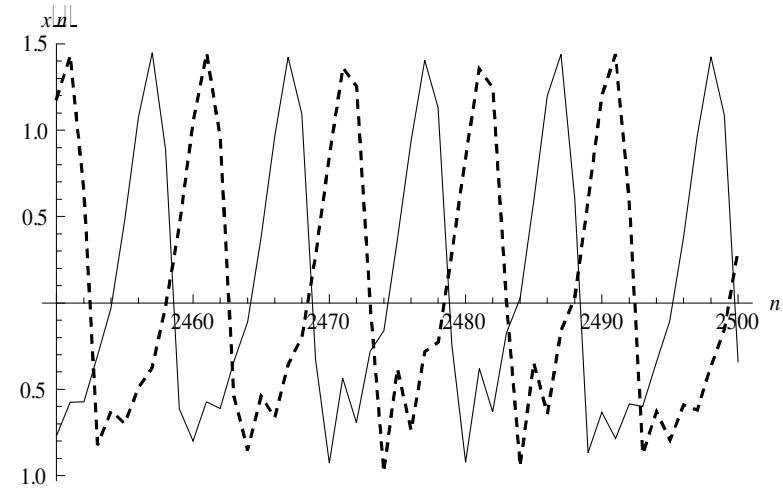

Fig-1: x-time series plot for $a=1, b=0.6$

\section{QUASI-PERIODIC ROUTE TO CHAOS:}

In the quasi-periodic scenario, the systems begins with a limit cycle trajectory. As the control parameter is changed, a second periodicity appears in the behavior of the system. This bifurcation event is the generalization of Hopf bifurcation. So, it is also called a Hopf bifurcation. In terms of characteristic multipliers, the Hopf bifurcation is marked by having the two complex-conjugate multipliers cross the unit circle simultaneously [21].

At the parameter value $a=0.3$, the two complex conjugate eigen-values at the fixed point $x_{1}^{*}=\frac{(a-1)+\sqrt{(1-a)^{2}+4 b}}{2}$ assumes the magnitude one. For $a<0.3$, the magnitude of the same remains less than one but as soon as $a$ crosses the value $a=0.3$, the magnitude becomes greater than one and this confirms the occurrence of Hopf bifurcation at $a=0.3$ and in turn confirms the quasi-periodic behavior which begins at $a=0.3$.

The quasi-periodic behavior which to the eye appear to be very similar to the chaotic behavior can be identified using the concept of Lyapunov Exponent. For periodic behavior the maximal lyapunov exponent (LMax) is negative, for quasiperiodic behavior LMax is zero and for the chaotic behavior LMax is positive quantity. But lyapunov exponent is the estimation of the exponential divergence of the trajectories when the number of iterations tends to $\infty$. and it is not possible to estimate it numerically using computer algorithm to find the limit values and hence we use large number of iterations to estimate LMax. In the table below we enlist the value of LMax for different number of iterationsat the parameter value $a=0.3$.
Table-1

\begin{tabular}{|l|l|}
\hline No. of iterations & Value of LMax \\
\hline 5000 & -0.000764118 \\
\hline 10000 & -0.000464176 \\
\hline 15000 & -0.000351312 \\
\hline 20000 & -0.000225448 \\
\hline
\end{tabular}

From the above table, it is found that LMax increases as the number of iterations increase and the values converge towards the value 0 , which ensures that the map exhibits quasiperiodic behavior at $a=0.3$. For $a=0.2$, LMax is found to be-0.029162 which is negative and is an indication of periodic behavior. For $a=0.96$, LMax is found to be 0.0305744 which is positive and indicates the chaotic behavior. Below we have shown the "LMax Vs the bifurcation parameter" diagram in steps of 0.02 .

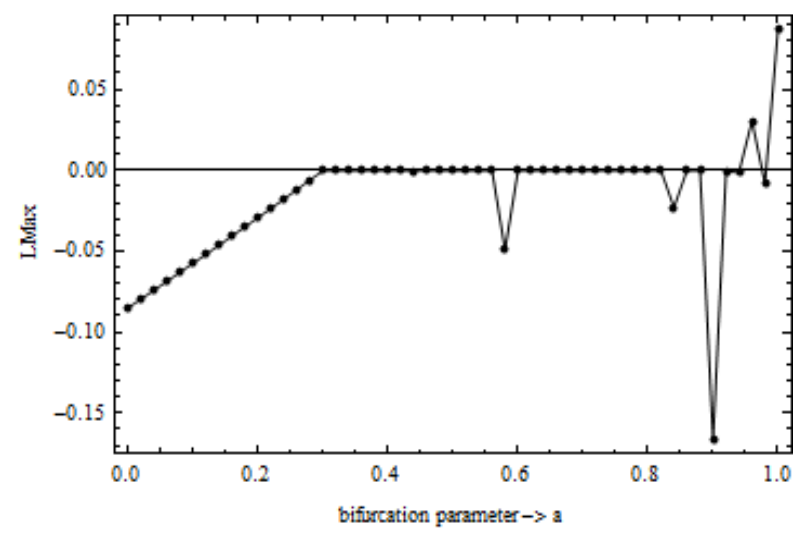

Fig-2: LMax Vs. Parameter “a”

Thus from the above discussion it is again confirmed that our map follows the quasi-periodic route towards chaos. This route can also be verified by the bifurcation diagram, phaseportrait, time-series plot, fourier-spectra, new-indicator diagram.

\subsection{Bifurcation diagram and attractor or phase-portrait :}

Below, we have shown the bifurcation diagram for $b=0.6$. Here the value of $a$ goes from 0 to 1 . Further, we have drawn the attractor for the map at the parameter values $b=0.6$ and $a=0.5$. 


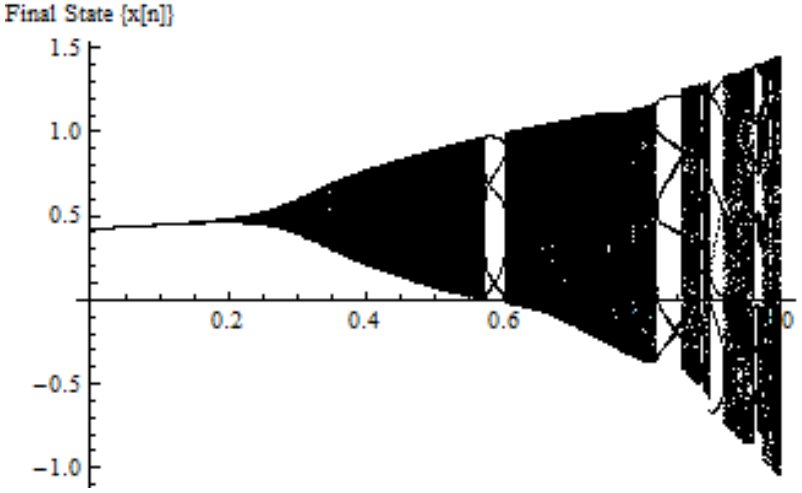

Fig 3: Bifurcation diagram of the map for $b=0.6$

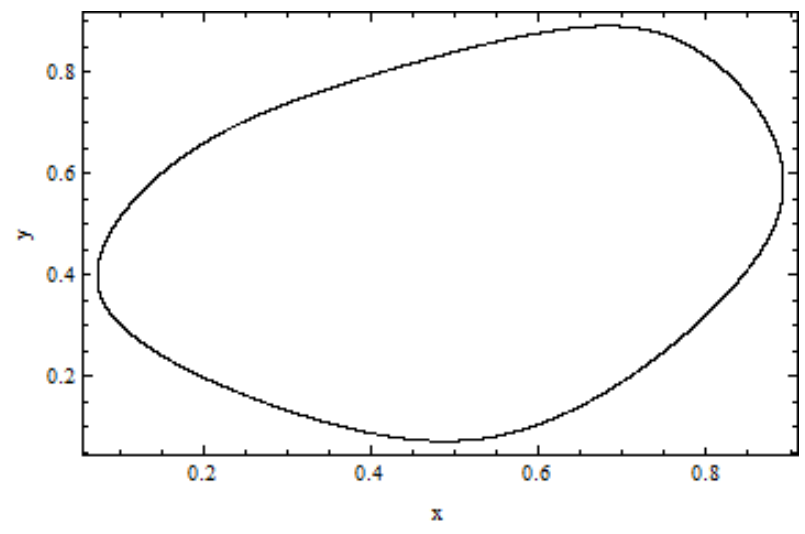

Fig-4: Phase-portrait for $b=0.6$ and $a=0.5$

The diagrams reflect the quasi-periodic behavior because in a quasi-periodic case, the attractor looks like a closed loop but actually the loop is disconnected which can be seen in this case by enlarging the above figure. The important point is that the attractor for the system is a two dimensional surface of a torus for quasi-periodic behavior [21]

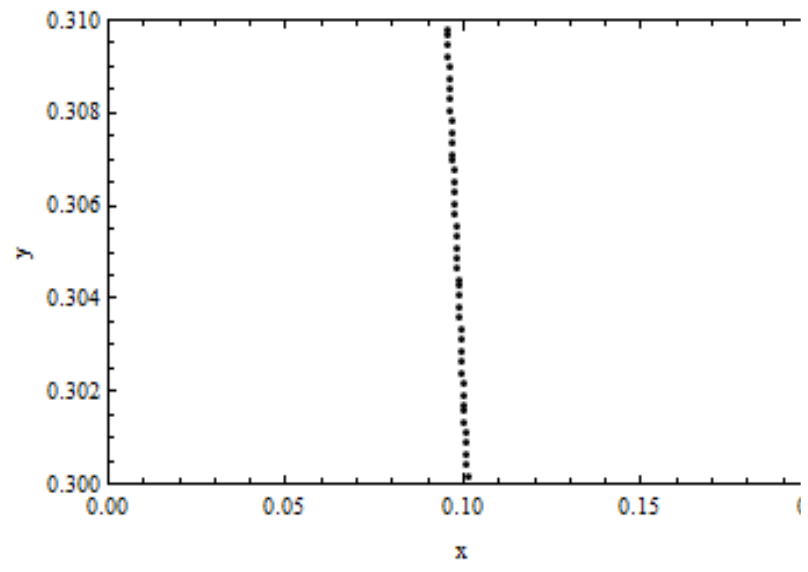

Fig-5: Enlargement of fig-4 showing disconnected loop

\subsection{Time series and Fourier Spectra:}

Below we have shown the $\mathrm{x}$-time series for $b=0.6$ and $a=0.5$. From the $\mathrm{x}$-time series plot it is observed that the system is not exactly periodic but close to periodic which is quasi-periodic. Further, we have shown the Fourier spectra which also substantiate our claim.

Fourier analysis lets us determine the frequency content of some signal. If the signal is periodic or quasi-periodic, then the Fourier spectra will consist of a sequence of "spikes" at the fundamental frequencies, their harmonics and the frequencies that are the sums and differences of various frequencies. The crucial point is that the spectrum will consist of a discrete set of frequencies. However, if the signal is non-periodic (for example, chaotic), then the Fourier spectra will be continuous. Thus, the sudden appearance of a continuous power spectra from a discrete spectrum, as some parameter of the system is changed, is viewed as an indicator of the onset of chaotic behavior [21].

Fourier analysis is a powerful tool which indicates the main (or all) frequency (-ies) from which a periodic time series (i.e. function, signal, trajectory) consists of. A Fourier spectrum is a diagram, whose horizontal axis represents the frequency and the vertical one the amplitude of each of the frequencies. In fact, a Fourier spectrum decomposes a time series in its component frequencies and shows signature at one (or more) fundamental frequency and at integer multiple (harmonics) of that, which may be infinite. In the case that our signal is periodic, its Fourier spectrum will consist of one fundamental frequency and all the harmonics of it. When the signal is quasi-periodic its spectrum will consist of two (at least) fundamental frequencies, whose ratio is an irrational number, and all of their harmonics. So, in these two cases spectrum will consist of discrete signatures. When the signal is chaotic the spectrum will show signatures at more than one fundamental frequencies and moreover a continuous background will emerge in the diagram.

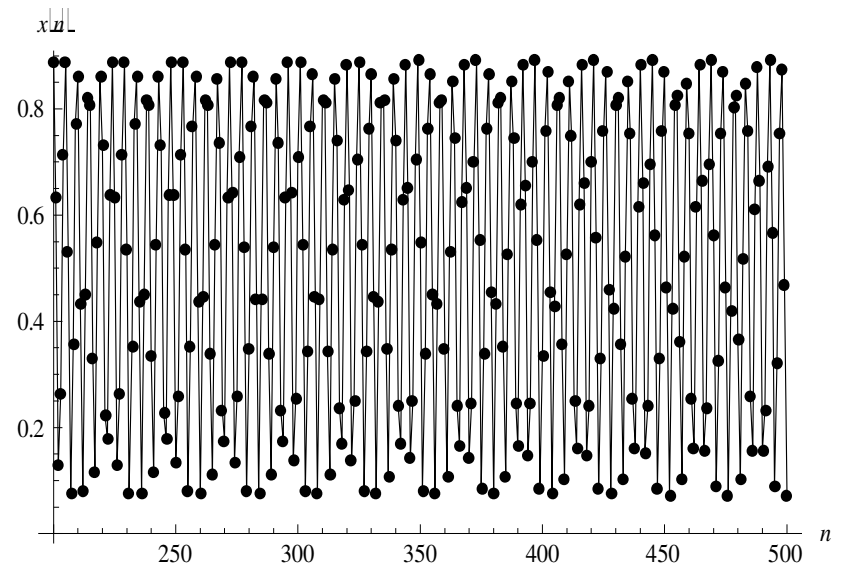

Fig-6: x-time series for $a=0.5, b=0.6$ showing the quasiperiodic behavior 


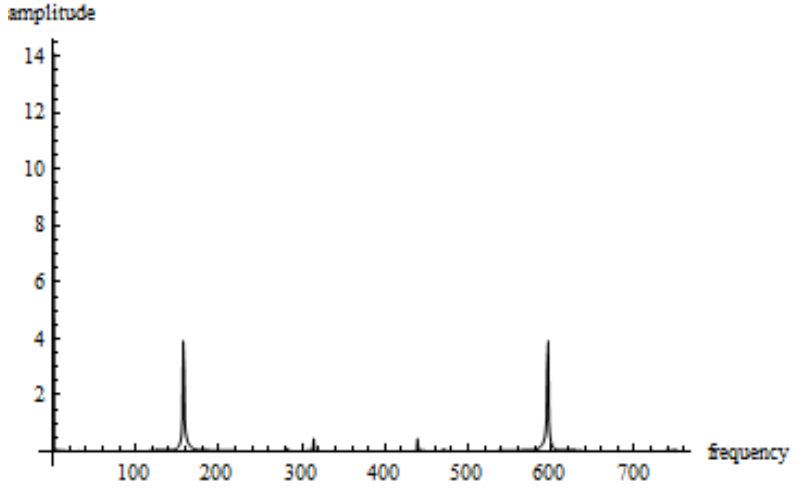

Fig-7: Fourier spectrum for $a=0.5, b=0.6$ corresponding to Quasi-periodic behavior

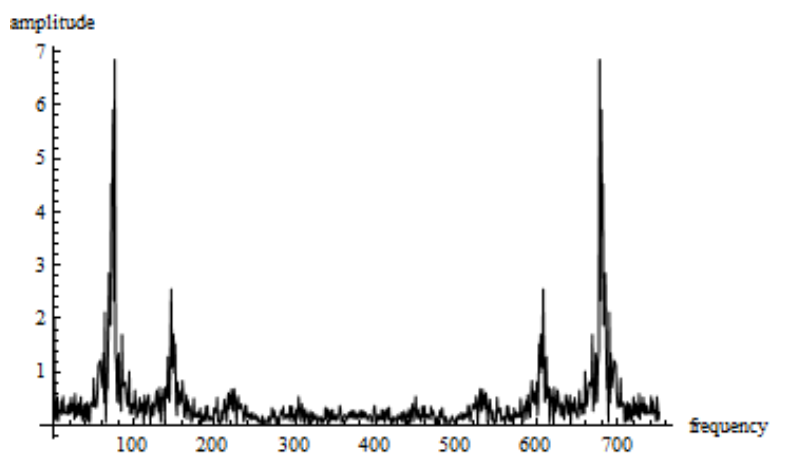

Fig-8: Fourier spectrum for $a=1, b=0.6$ corresponding to Chaotic behavior

\subsection{A proposed New indicator diagram [13] to show periodic, Quasi-periodic and} Chaotic behavior :

A well known route to chaos is the quasi-periodic route which was proposed by Ruelle and Takens 1971; Kaneko 1986, [2, $9,19,21]$ where the dynamics of the system initially undergo a bifurcation from a stable equilibrium point into periodic oscillations. As the control parameter is varied further, successive (Hopf) bifurcations introduce new fundamental frequencies to the motion. If these frequencies are incommensurate, quasi-periodic fluctuations are obtained, where the trajectory densely cover the surface of the attractor in the phase space, in this case referred to a torus. Otherwise, frequency locking is observed where the trajectories close after a finite number of cycles. Further variations in the control parameter cause perturbations that drive the system into chaos, breaking down the torus into strange attractor. For this reason, the route is also referred to as the torus breakdown route [19]

Below, we have shown the attractor for $b=0.6$ and $a=1$ To show that the above attractor is chaotic, we plot the proposed new indicator diagram [13], where we plot the largest eigen-value in magnitude Vs. the iteration number.

From the proposed new indicator diagram it is observed that the eigen-values are arbitrarily placed which assures that the system is chaotic for $b=0.6$ and $a=1$.
We can use the proposed new indicator diagram to show quasi-periodic behavior also which ascertains that our map follows the quasi-periodic route to chaos also.

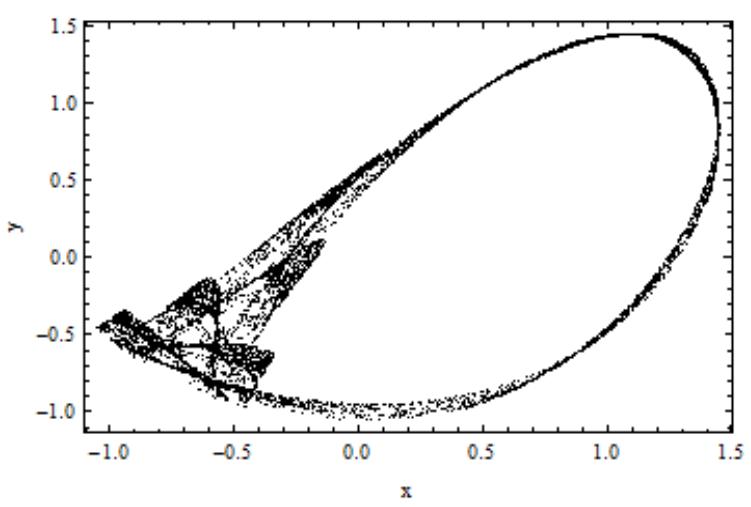

Fig-9: Attractor for $b=0.6$ and $a=1$

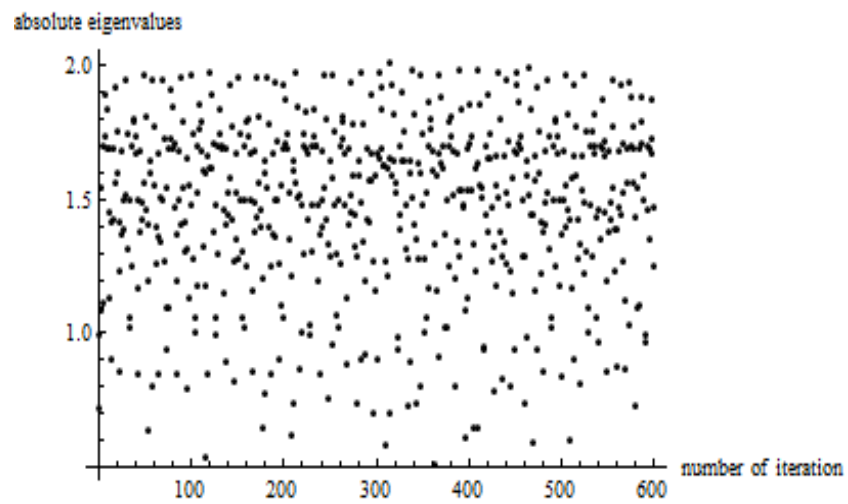

Fig-10: Proposed New indicator diagram for $b=0.6, a=1$ showing chaotic nature

\section{THE PERIODIC PROPORTIONAL PULSE METHOD TO CONTROL CHAOS IN TWO-DIMENSIONAL DISCRETE MAP:}

Chaotic region for a system may contain many possible orbits, being able to select between several of these by changing control parameters allows multiple responses from the same system. Thus, it is often useful to stabilize a chaotic orbit to one that is regular and predictable. Chaos control explores the intrinsic richness of the chaotic behavior by stabilizing unstable periodic orbits embedded in a chaotic attractor. Controlling chaos by various methods are well studied in $[5$, $6,7,11,22,23,24,26]$.

In $[12,15]$, the authors introduced a specific method to have control over chaos. In discrete dynamics, the authors applied instantaneous pulses on the system variables $X_{i}$, once every $p$ iterations, in the form

$X_{i}=k X_{i} \quad(\mathrm{i}$ is a multiple of $p)$

where $k$ is a constant. In the case of continuous dynamics, the pulses are to be applied to variable $X(t)$, at regular instants $p \tau$ $(\tau=$ constant $)$. 
In [18], the author re-examined the control of the form (1) in more detailed form. In two-dimensional map, the control technique is described as follows:

Consider a two-dimensional discrete system,

$$
\begin{aligned}
& x_{n+1}=f\left(x_{n}, y_{n}\right), y_{n+1}=g\left(x_{n}, y_{n}\right) \\
\text { or, } \quad X_{n+1} & =F\left(X_{n}\right)
\end{aligned}
$$

where $X$ is a vector in $\Re^{2}$ and $\mathrm{F}$ is a map of a domain $D \subseteq \Re^{2}$ onto itself.

To control the dynamics, one needs to apply kick to its orbit once every $p$-steps, by multiplying the $\mathrm{x}$-component of the dynamics by a factor $k_{1}$ and the y-component by a factor $k_{2}$. The kicked map is defined as $G=K F^{p}$, where $K$ is a diagonal matrix having the diagonal elements as $k_{1}$ and $k_{2}$.

A fixed point $X_{s}$ of $G$ satisfies the equation,

$$
K F^{p}\left(X_{s}\right)=X_{s}
$$

This fixed point $X_{S}$ is locally stable if,

The Jacobian of $G$ has two eigenvalues $<1$ in magnitude. (5)

Controlling the dynamics means to find appropriate values of $k_{1}$ and $k_{2}$ so that the kicked dynamics is stabilized at a periodic orbit of period- $p$. For a given period $p$ and a given point $X_{s}$, the constants $k_{1}, k_{2}$, can be derived from (4), and the problem is to test if, with these kicking factors, the condition (5) is satisfied.

\subsection{Controlling chaos in our considered map:}

Our considered map is, $x_{n+1}=y_{n}, y_{n+1}=a y_{n}+b-x_{n}^{2}$ or $X_{n+1}=F\left(X_{n}\right)$

where $F(x, y)=\left(y, a y+b-x^{2}\right)$.

This map exhibits chaotic behaviour for $a=1, b=0.6$. We controlled the map at these parameter values by stabilizing the orbit to a periodic point of period-1(i.e. we considered $p=1$ ) as follows:

Since $p=1$, so we have $G=K F=\left(k_{1} y, k_{2}\left(a y+b-x^{2}\right)\right)$

We considered the point $X_{s}=\left(x_{s}, y_{s}\right)=(0.1,0.5)$, then the Jacobian of $\mathrm{G}$ at the point $X_{s}=\left(x_{s}, y_{s}\right)$ is given by

$G_{1}=\mid$ Jacobian $\left.G\right|_{X=X_{s}}=\left[\begin{array}{cc}0 & k_{1} \\ -2 k_{2} x_{s} & k_{2} a\end{array}\right]=\left[\begin{array}{cc}0 & k_{1} \\ -0.2 k_{2} & k_{2}\end{array}\right]$

The eigenvalues of $G_{1}$ are given by

$\lambda_{ \pm}=\frac{k_{2} \pm \sqrt{k_{2}^{2}-0.8 k_{1} k_{2}}}{2}$

Again (4) becomes, $K F\left(X_{S}\right)=X_{S}$ which after calculation gives the values of $k_{1}=0.2$ and $k_{2}=0.45816$. With these values of $k_{1}$ and $k_{2}$, using (6) we found the eigenvalues of $G_{1}$ as 0.413881 and 0.0442794 , both of which are less than one in magnitude and hence we are done.

Below, we have shown the plot of the time series data for the original map with and without control. Here we have activated the control at 100th iterate and switched it off at the 200th iterate. We also have plotted the new-indicator diagram and attractor for the kicked map at the prescribed parameter values.

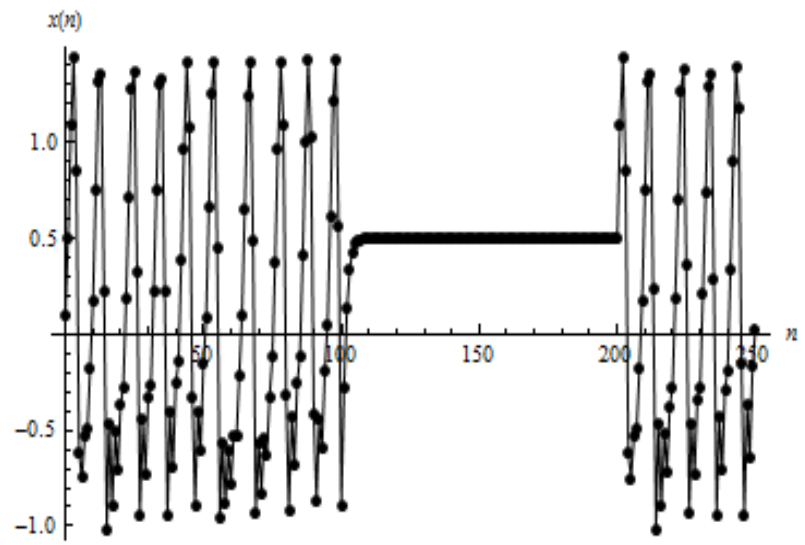

Fig-12: Time series data with and without control (for $b=0.6, a=1$ )

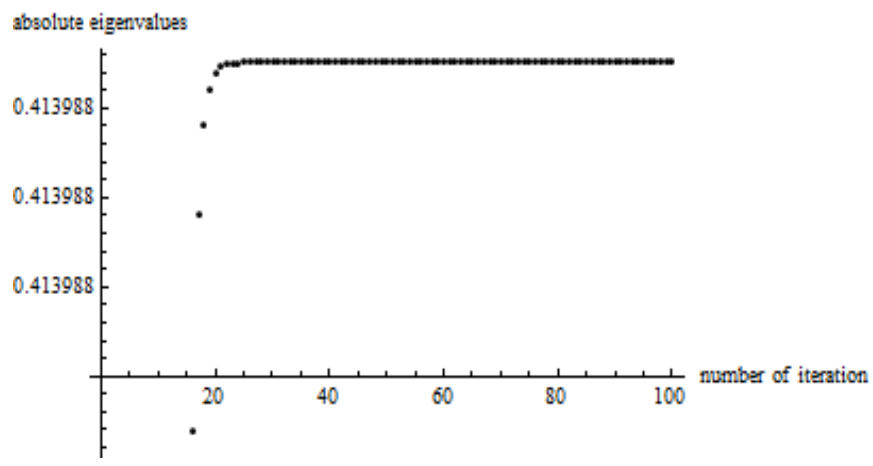

Fig 13: New-indicator diagram for the kicked or the controlled map showing a regular pattern i.e. periodic behaviour.

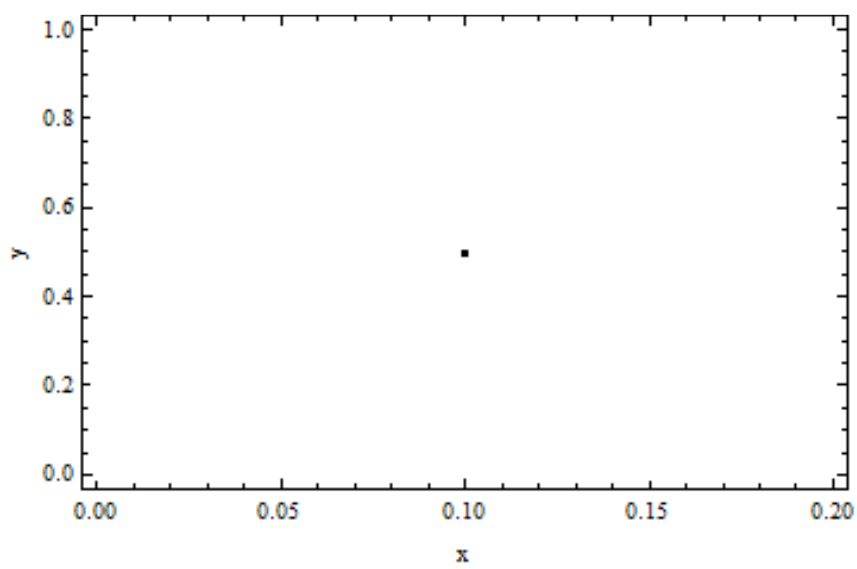

Fig 14: Attractor for the kicked map showing period-1 behavior

\section{CORRELATION DIMENSION VS. EMBEDDING DIMENSION:}

The goal of determining the dimension of an attractor is that the dimensionality of an attractor furnishes information on 
the number of dominant variables present in the evolution of the corresponding dynamical system. A finite, non-integer value of the dimension is considered to be an indication of the presence of chaos [21].

The definition of box-counting dimension is conceptually straight forward but it is very difficult to compute practically as the number of computations required for the boxcounting procedure increases exponentially with the state space dimension. Moreover, the box-counting scheme requires us to partition the state space with boxes and then to locate the trajectory points within the boxes which is a very time consuming process. So to provide a computationally simpler dimension for an attractor, Grassberger and Procaccia introduced a dimension based on the behaviour of a so-called correlation sum (or correlation integral) $[2,4,9]$. This dimension is called the correlation dimension and has been widely used to characterize chaotic attractors. It has a computational advantage because it uses trajectory points directly and does not require a separate partitioning of the state space.

For a d-dimensional phase space the correlation function is

$$
C^{d}(R)=\lim _{N \rightarrow \infty} \frac{2}{N(N-1)} \sum_{i, j=1, i \neq j}^{N} H\left(R-\left|Y_{i}-Y_{j}\right|\right)
$$

where $\mathrm{H}$ is the Heaviside step function with $\mathrm{H}(\mathrm{x})=1$ for $x>0$ and $H(x)=0$ for $x \leq 0, N$ is the number of points on the reconstructed attractor and $\mathrm{R}$ is the radius of the sphere centered on $Y_{i}$ or $Y_{j}$.

The correlation function $C^{d}(R)$ is related to the radius $\mathrm{R}$ by the relation

$$
C^{d}(R)=m R^{-D_{c}(d)}
$$

Where $D_{c}(d)$ is the correlation dimension which is the slope of the $\log C^{d}(R)$ verses $\log R$ plot. That is,

$$
D_{c}(d)=\lim _{R \rightarrow 0, N \rightarrow \infty} \frac{\log C^{d}(R)}{\log R}
$$

Here we have calculated the correlation dimensions of the map for various embedding dimensions at the parameter values $b=0.6, a=1$ and then plotted the graph of correlation Vs embedding dimension. It is seen that the correlation dimension is more or less equal to 1.2 (noninteger) for almost all embedding dimensions which indicates the presence of chaotic attractor [14]

Table: 2

\begin{tabular}{|l|l|}
\hline Embedding dimension & Correlation dimension \\
\hline 1 & 0.682354 \\
\hline 2 & 1.03559 \\
\hline 3 & 1.12072 \\
\hline 4 & 1.16714 \\
\hline 5 & 1.17923 \\
\hline 6 & 1.19052 \\
\hline 7 & 1.20511 \\
\hline
\end{tabular}

\begin{tabular}{|l|l|}
\hline 8 & 1.20719 \\
\hline 9 & 1.21716 \\
\hline 10 & 1.24276 \\
\hline
\end{tabular}

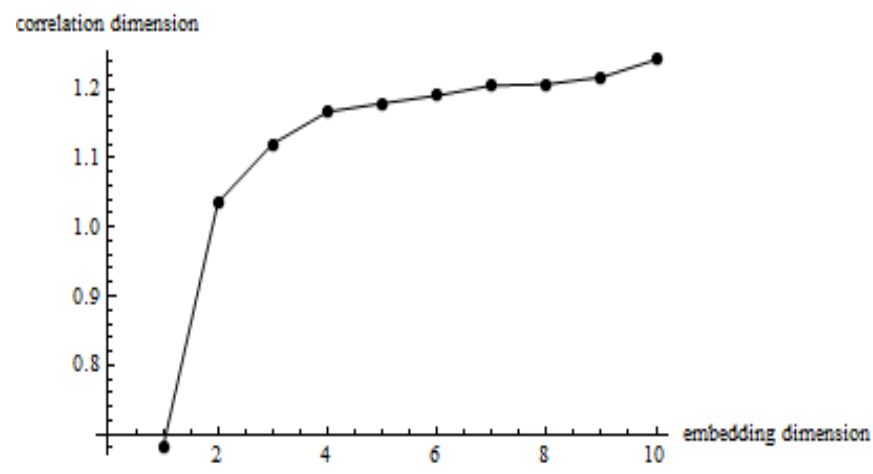

Fig-15: Correlation dimension Vs Embedding dimension at $b=0.6, a=1$

\section{MAP WITH SLIGHT MODIFICATION:}

Considering the map $W^{\prime \prime}(x, y)=\left(|y|,\left|a y+b-x^{\wedge} 2\right|\right)$ i.e. considering the absolute values of both the populations, it is observed that this system reaches chaos through a mixture of universal routes (quasi-periodic, period doubling and period un-doubling route). Thus a slight change in the map changes the route to chaos. Moreover chaos occurs earlier in this modified system than the original map. In the original map chaos come into existence after the parameter value 0.95 but in this modified map chaos can be observed before the parameter value 0.9 . This modified map is also more practical as because we are dealing with population.

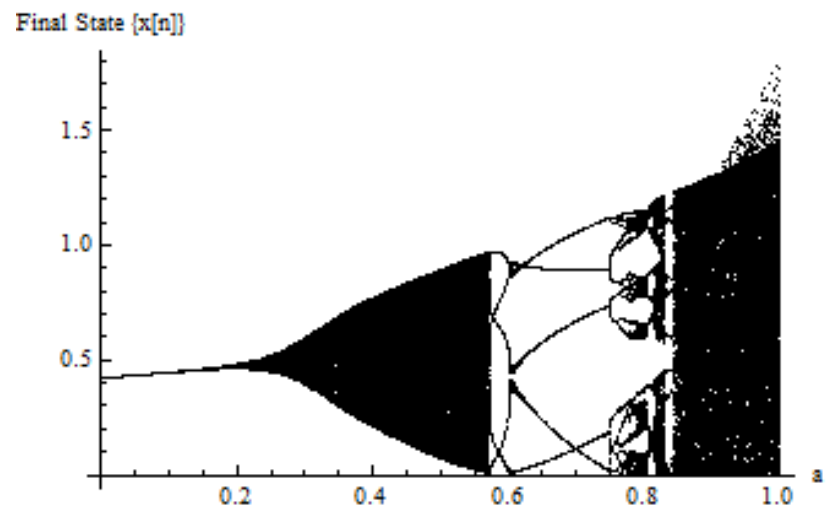

Fig-16: Bif. Diagram for the modified map for $b=0.6, a=1$ 


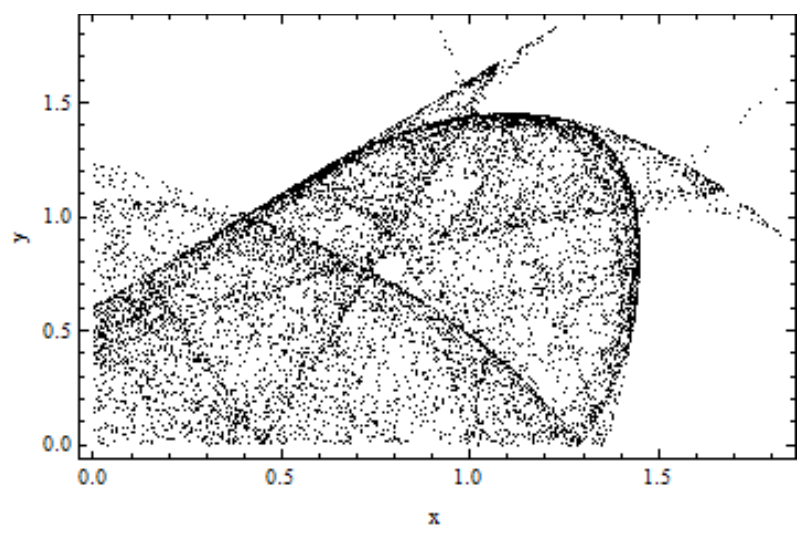

Fig-17: Phase plot for the modified map for $b=0.6, a=1$

\section{REFERENCES}

[1] B. V. Chirikov, A universal instability of manydimensional oscillator systems, Phys. Rep. 52, 263 (1979).

[2] B. L. Hao, Chaos 2, World Scientific, Singapore, 1990.

[3] D. Whitley, On the Periodic Points of a Two -Parameter Family of Maps of the Plane, Acta Applicandae Mathematicae 5 (1986), pp 279-311

[4] D. G. Aronson, M. A. Chory, G. R. Hall, and R. P. McGehee : Bifurcations from an Invariant Circle for Two-Parameter Families of Maps of the Plane : A Computer Assisted Study, Commun. Math. Phys. 83 (1982), pp 303-354

[5] E. Ott, C. Grebogi and J. A. Yorke "Controlling Chaos", Phys. Rev. Lett. 64, (1990), pp 1196-1199

[6] E. Ott, "Controlling chaos", Physics Today, Vol. 48, Issue 5, (1995) pp 34-40.

[7] F. J. Romeiras, "Controlling Chaotic Dynamical Systems”, Physica D, vol. 58, (1992) pp 165-192,

[8] G. Casati and B. V. Chirikov, Quantum Chaos (Cambridge Univ. Press, 1995),

[9] H. G. Schuster, Deterministic Chaos (Physik-Verlag, Weinheim 1984),

[10] J. Guckenheimer, G. Oster, and A. Ipatchki, : The Dynamics of Density Dependent Population Models, J. Math. Biol. 4 (1977), pp 101-147
[11] J. Singer, "Controlling a Chaotic System", Physical Review Leters, vol 66, (1991) pp 1230-1232,

[12] J. Guemez and M.A. Matias, Control of chaos in unidimensional maps, Physics Letters A 181, (1993) pp 29-32.

[13] L. M. Saha and M. Budhraja, The Largest Eigenvalue : An indicator of Chaos? Int. J. of Appl. Math and Mech. 3(1) (2007) pp 61-71.

[14] Maria I. Loffredo : Testing chaos and fractal properties in economic time series,www.internationalmathematicasymposium.org/IM S99/paper25/ims99.

[15] M.A. Matias and J. Guemez, Stabilization of chaos by proportional pulses in system variables, Physical Review Letters 72, (1994), pp 1455-1458.

[16] M. J. Feigenbaum, "The Universal Metric Properties of Nonlinear Transformations." J. Stat. Phys. 21, 669-706, 1979.

[17] M. J. Smith : Mathematical Ideas in Biology, CambridgeUniv. Press, 1968

[18] N.P. Chau, Controlling chaos by periodic proportional pulses, Phys. Lett. A, 234 (1997), pp 193-197

[19] P. Rohani, O. Miramontes and M. P. Hassell, Quasiperiodicity and chaos in population models, Proc. R. Soc. Lond. B (1994) 258, pp 17-22

[20] R. M. May : Biological Populations Obeying Difference Equations : Stable Points, Periodic Orbits and Chaos, J. Theor Biol, 51 (1975), pp 511-524

[21] R. C. Hilborn, Chaos and nonlinear dynamics, Oxford University Press 1994

[22] T. Kapitaniak, Controlling Chaos : Theoretical and Practical Methods in Non-Linear Dynamics, Academic Press, New York, 1996

[23] T. Shinbrot, "Using Small perturbations to Control Chaos", Nature, Vol. 363 (1993) pp 411-417.

[24] W. L. Ditto, "Experimental control of Chaos", Physical Review Letters, vol 65, No. 26, (1990) pp 3211-3214,

[25] Y. T. Li and J. A. Yorke : "Period Three Implies Chaos", Amer. Math. Monthly 82 (1975), pp 985-992

[26] Z. Gills, "Tracking Unstable Steady States: Extending the Stability Regime of a Multimode Lazer System", Physical Review Letters, vol. 69, No. 22, (1992) pp 3169-3172, 